Portland State University

PDXScholar

1978

\title{
Intelligence and nonintelligence factors contributing to scores on the Rorschach prognostic rating scale
}

Anneke P. Hathaway

Portland State University

Follow this and additional works at: https://pdxscholar.library.pdx.edu/open_access_etds

Part of the Cognition and Perception Commons

Let us know how access to this document benefits you.

\section{Recommended Citation}

Hathaway, Anneke P., "Intelligence and nonintelligence factors contributing to scores on the Rorschach prognostic rating scale" (1978). Dissertations and Theses. Paper 2887.

https://doi.org/10.15760/etd.2881

This Thesis is brought to you for free and open access. It has been accepted for inclusion in Dissertations and Theses by an authorized administrator of PDXScholar. Please contact us if we can make this document more accessible: pdxscholar@pdx.edu. 
AN ABSTRACT OF THE THESIS OF Anneke P. Hathaway for the Master of Arts in Psychology presented December 12, 1978.

Title: Intelligence and Nonintelligence Factors Contributing to Scores on the Rorschach Prognostic Rating Scale.

APPROVED BY MEMBERS OF THE THESIS COMMITTEE :

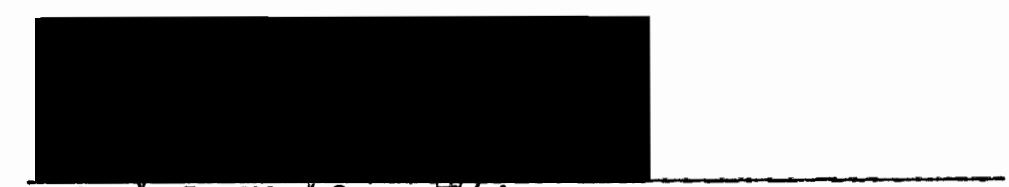

Walter G. Klopfer, OKair
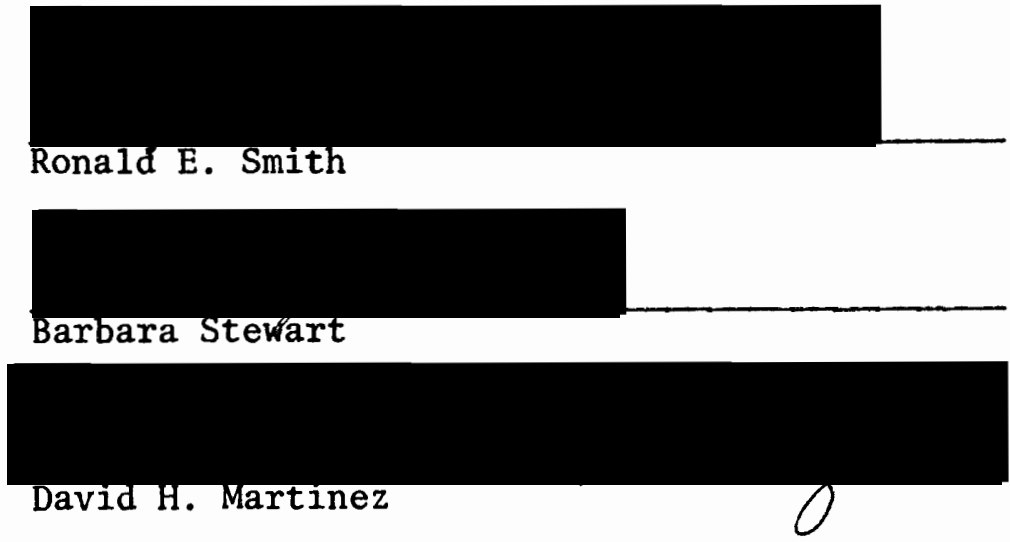

Correlation between the RPRS total and WAIS FSIQ showed that approximately $43 \%$ of the variance in the RPRS total can be accounted for by the variance in the FSIQ. Verbal IQ is not significantly higher correlated with the RPRS total than the Performance IQ $(p<.10)$. Factor analysis of the 6 RPRS subscores resulted in 2 factors. Subsequent factor analysis of the 6 RPRS subscores plus FSIQ and the factor 
analysis of the 6 RPRS subscores plus PIQ and VIQ showed the first factor to be an intelligence factor accounting for $85 \%$ of the common variance. The RPRS variables FL, FM, M and Sh loaded on this factor. The second factor was a nonintelligence factor accounting for $15 \%$ of the common variance. Small $\mathrm{m}$ is clearly the main determinant of this factor followed by Sh. In addition, the results of 2 exploratory factor analyses are discussed. The first exploratory factor analysis involved 23 variables, namely the 6 RPRS subscores, RPRS total, the 11 WAIS subscales, FSIQ, VIQ, PIQ, VIS and OABD. The second exploratory analysis involved 17 variables, namely the 6 RPRS subscores and the 11 WAIS subscales. 
INTELLIGENCE AND NONINTELLIGENCE FACTORS

CONTRIBUTING TO SCORES

ON THE RORSCHACH PROGNOSTIC RATING SCALE

by

ANNEKE P. HATHAINAY

A thesis submitted in partial fulfillment of the requirements for the degree of

MASTER OF ARTS

in

PSYCHOLOGY

Portland State University 
TO THE OFFICE OF GRADUATE STUDIES AND RESEARCH:

The members of the Committee approve the thesis of Anneke P. Hathaway presented December 12, 1978.

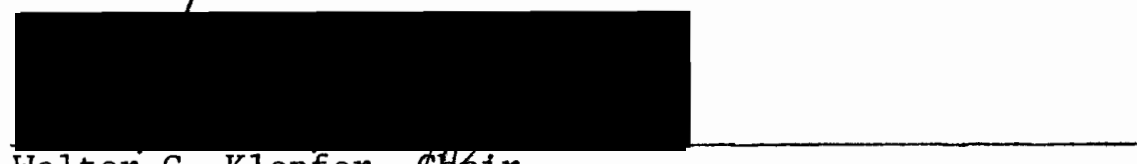

Walter G. Klopfer, GHair

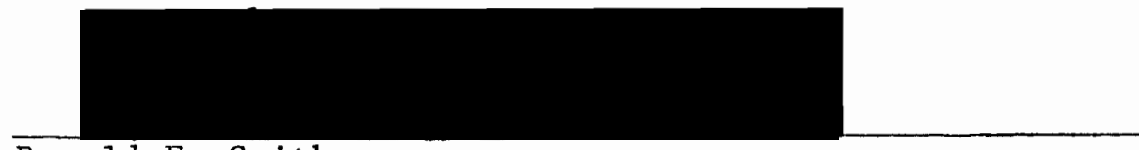

Ronald E. Smith

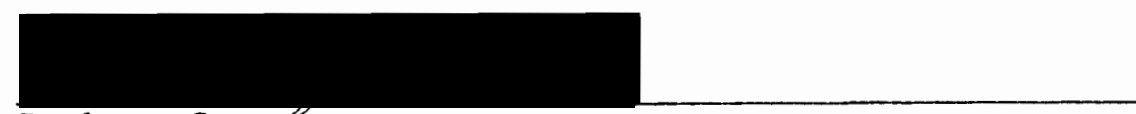

Barbara Stewät

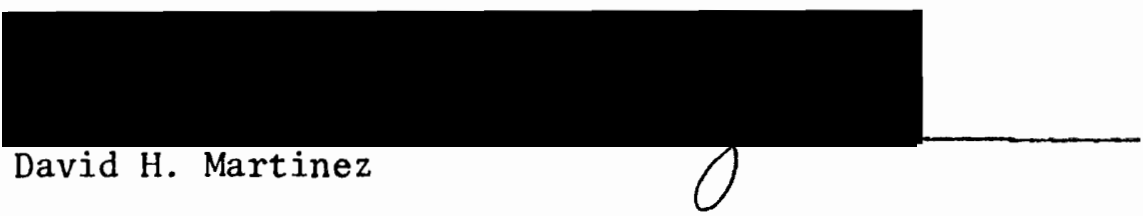

APPROVED :

Robert E. Jones, Jr., Head, Department of Psychology

Stanley E. Rauch, Dean of Graduate Studies and Research 


\section{ACKNOWLEDGMENTS}

I wish to first thank my husband for the support and encouragement he has provided, especially as I have made the transition from the European to the American academic world. But that is only a small part of his contribution. He is the person most intimately related to my personal and scientific quest.

Dr. Walter Klopfer has been the person most directly helpful along the path of my recent intellectual search. His enthusiasm for his work is contagious to many of his students, including me, and the results of that work form the basis for this study.

Barbara Mecca has been not only a good personal friend, but the source of many protocols. Dr. John Exner from the Rorschach Workshops also heeded my plea for data. Without his help I would still be collecting data. Dr. Barbara Stewart's thoroughness, competence and interest was as invaluable to me as it is to many other graduate students. Dr. Ronald Smith not only introduced me to Intelligence Tests but has given me the support and encouragement needed for perusing research in this area. John Garwood is the only person who could have been such a patient and competent independent rater.

Mrs. Marguerite Spring put this study in a presentable form. Her editorial and typing skills have been indispensable to me.

Finally, I extend my appreciation to Sigma Xi, the Scientific Research Society of North America, who provided funds to partially. support this study through a frant-in-Aid Research. 
TABLE OF CONTENTS

PAGE

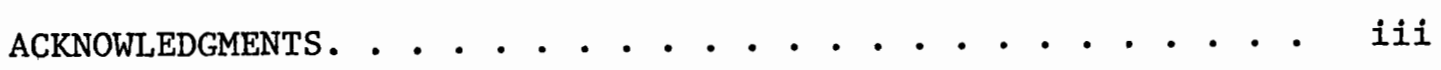

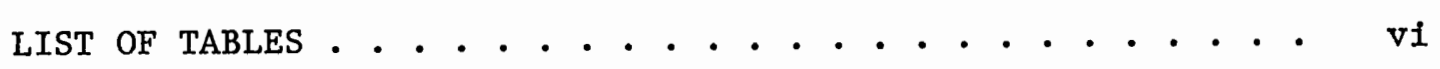

CHAPTER

I INTRODUCTION . . . . . . . . . . . . . . . 1

II THE RORSCHACH PROGNOSTIC RATING SCALE (RPRS) . . . 3

History. • • . . . . . . . . . . . . 3

Calculating the RPRS . . . . . . . . . . 3

The prognostic validity of the RPRS. . . . . . 6

III INTELLIGENCE AND SUCCESS IN PSYCHOTHERAPY. . . . . . 10

A summary of the research. . . . . . . . . 10

IV IQ AND THE RPRS. . . . . . . . . . . . . . . . . 13

A summary of the research. . . . . . . . . 13

V METHOD . . . . . . . . . . . . . . . . . 16

Introduction . . . . . . . . . . . . 16

Sample Descriptions. . . . . . . . . . 21

Data Collection. . . . . . . . . . . . 21

Analytic Procedures. . . . . . . . . . . 22

Hypotheses . . . . . . . . . . . . . . 23

Significance . . . . . . . . . . . . . 25 
v

CHAPTER

PAGE

$$
\begin{aligned}
& \text { VI RESULTS. . . . . . . . . . . . . . } 28 \\
& \text { Descriptive Statistics . . . . . . . } 28 \\
& \text { Correlations ............. . } 29 \\
& \text { Factor Analysis. . . . . . . . . . } 31 \\
& \text { VII CONCLUSIONS. . . . . . . . . . . . . . } 37 \\
& \text { Conclusions about the Hypotheses . . . . . . } 37 \\
& \text { Conclusions about the Exploratory Factor } \\
& \text { Analyses . . . . . . . . . . . . . } 40
\end{aligned}
$$

VIII SUMMARY AND RECOMMENDATIONS FOR FUTURE RESEARCH. . . . . . . . . . . . . . . 46

BIBLIOGRAPHY . . . . . . . . . . . . . . . . . 50 


\section{LIST OF TABLES}

TABLE

PAGE

I Studies on the Relationship to RPRS Subscores and

Succeśs in Psychotherapy. . . . . . . . . .

II Studies on Psychotherapy and Intelligence . . . . . . 11

III Studies of Relationship of IQ and RPRS. . . . . . . . . 14

IV Variables . . . . . . . . . . . . . . . . 17

$\mathrm{V}$ Means and Standard Deviations for WAIS and

RPRS Variables. . . . . . . . . . . . 18

VI Correlation Table . . . . . . . . . . . . . 19

$\mathrm{VI}^{\mathrm{A}}$ Intercorrelation of WAIS Subscales. . . . . . . . . . 20

VII Factor Matrix of the 6 RPRS Subscales . . . . . . . 32

VIII Factor Matrix of the 6 RPRS Subscales and FSIQ. . . . . 33

IX Factor Matrix of the 6 RPRS Subscales, VIQ and PIQ. . . 34

X Factor Matrix of all 23 Variables . . . . . . . . 35

XI Factor Matrix of the 6 RPRS Subscales and 11 WAIS

Subscales . . . . . . . . . . . . 36 


\section{CHAPTER I}

\section{INTRODUCTION}

As the demand for psychological services grows psychologists are increasingly required to screen and select candidates for psychotherapy. One major criterion applied in making such difficult decisions is the projection of a successful outcome from the proposed therapy. As Garfield and Affleck (1961) have indicated, this prognosis is often based on intuitive and subjective criteria. They found that therapists' rating of prognosis is highly correlated with the personal feelings of the raters towards the patients $(r=.66 ; p<.05)$. Ratings of "interest in taking patient into treatment" were also highly correlated with both rating of therapeutic prognosis $(r=.65 ; p<.05)$ and the personal feelings of the raters towards the patient $(r=.63$; $\mathrm{p}<.05)$. They conclude that "the average therapist prefers a patient who is intelligent, well motivated for therapy, young and with some insight into his difficulties." This description bears close resemblance to the type of preferred patient mentioned in the research by Hollingshead and Redlich (1958). It is highly likely that a patient with such attributes will benefit from psychotherapy, but what about the person who does not quite fit this description of "ideal client"? A more objective and scientific approach to identification and use of the factors which contribute to a favorable prognosis seems to be desirable. 
This study will attempt to help identify these factors, by means of a factor-analytic study of the Rorschach Prognostic Rating Scale (RPRS) and of the Wechsler Adult Intelligence Scale (WAIS). 
CHAPTER II

THE RORSCHACH PROGNOSTIC

RATING SCALE

\section{History}

The RPRS was developed by Bruno Klopfer and published in 1954 (Klopfer et al 1954). It was designed to use the Rorschach to predict a patient's response to psychotherapy and to establish treatment goals and treatment potential. The rating scale was conceived as a measure of the total ego-strength potential. It measures available ego-strength and the unused portion of adjustment potential which might be mobilized through psychotherapy. Both the used and unused portions of the ego-strength are measured together. The RPRS is especially useful for patients who are placed in a diagnostic category generally assumed to have unfavorable prognosis for therapy. The RPRS is able to distinguish those patients who have sufficient ego-strength to benefit from psychotherapy despite their diagnostic "label". In these cases there will be a discrepancy between clinical picture and ego-strength. This discrepancy will be diminished by successfu1 therapy (Klopfer et al 1954).

\section{Calculating the RPRS}

The RPRS, as described by Bruno Klopfer consists of six categories: 
1) Human movement (M) relates to the ability to deal with real human relations in an emphatic way.

2) Animal movement (FM) is an index of the handling of stress tolerance and the degree of comfort or lack of comfort a person feels with regard to his drive impulses.

3) Inanimate movement (m) indicates subjective discomfort.

4) Shading responses (Sh) show how affectionate needs are organized and expressed. These organized patterns range from insensitivity, evasion and denial of affectionate needs to full acceptance and the ability to enter into differentiated emotional relationships.

5) Color responses (C) shows the ability to respond emotionally to the outer world with adequate control.

6) Form level of the response (FL). High form level rating indicates the person's ability to have good reality contact.

All of the responses in each category are rated according to the criteria developed by Klopfer. For example, each $M$ response is rated according to the three criteria below and then the average of the three ratings is assigned to the response. This gives the M raw score.

Criteria

Rating

1) Amount of movement in space, described or implied

a) Increasing living space (dancing, running, talking together, pointing)

b) Decreasing living space (bowing, kneeling, crying, crouching, and all HD responses)

c) Merely alive (sleeping, lying down, sitting, balancing)

2) Freedom in seeing movement

a) Spontaneously sees action 1

b) Uses intermediary means of representing movement (picture of someone walking)

c) Reluctantly given in inquiry or follows only from the logic of the situation 
Criteria (cont.)

Rating

3) Cultural distance

a) Real people of immediate cultural milieu 1

b) Culturally distant real people; culturally popular fantasy figures; and figures whose clothing or equipment practically conceals their human form (Ubangis, Mickey Mouse, Superman, Diver in diving suit)

c) Unusual fantasy figures or culturally and/or historically extremely distant (Neanderthal man)

The average ratings of all the $M$ responses are added algebraically, counting each $M-$ response -1 . The resulting raw score is converted into a weighted score by the following table:

M raw score $\quad$ M weighted score

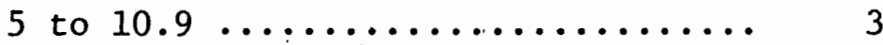

3 to 4.9 or 11 to $15.9 \ldots \ldots \ldots \ldots$. 2

1 to 2.9 or 16 to $20.0 \ldots \ldots \ldots \ldots$. 1

Less than 1 or more than $20.0 \ldots \ldots$. 0

Less than 0 (any minus score)...... - 1

Clues appropriate for the calculation of animal movement (FM), inanimate movement (m), shading ( $\mathrm{Sh}$ ), color (C), and form level (FL) are similarly employed, The sum of the weighted scores of the six Rorschach variables for each patient constitutes the final prognostic score:

\begin{tabular}{lll} 
Range & Group & \multicolumn{1}{c}{ Meaning } \\
17 to 13 & I & $\begin{array}{l}\text { The person is almost able to help himself. A } \\
\text { very promising case that just needs a little } \\
\text { help. }\end{array}$ \\
12 to 7 & II $\quad \begin{array}{l}\text { Not quite so capable as the above case to work } \\
\text { out his problems himself but with some help is } \\
\text { likely to do pretty well. } \\
\text { Better than 50-50 chance; any treatment will } \\
\text { be of some help. }\end{array}$
\end{tabular}




$\begin{array}{lrl}\text { Range } & \text { Group } \\ 1 \text { to }-2 & \text { IV } & \begin{array}{l}50-50 \text { chance. } \\ -3 \text { to }-6\end{array} \\ -7 \text { to }-12 & \text { VI } & \begin{array}{l}\text { A difficult case that may be helped somewhat } \\ \text { but is generally a poor treatment prospect. }\end{array} \\ & \text { A hopeless case. }\end{array}$

The prognostic validity of the RPRS

The research of the last 25 years on the prognostic validity of the RPRS :has been summarized by Garwood (1977). He concludes that the RPRS has proven to be an accurate measure of prognosis independent of type of therapy used. A total of 13 studies correlating RPRS total with therapeutic outcome were reported. Eight of these studies, involving 11 different client groups, report significant positive correlations with a successful outcome the correlations between RPRS and therapeutic outcome range from .38 to 80 . Five of these studies, on five different client groups, do not report a significant correlation. However, three of these studies report a non-significant positive correlation. One study does not report the sign of the correlation (Whiteley and Blaine, 1967). And in the Bloom (1956) study the sign of the correlation is negative for the underproductive group. The underproductive group is defined as having no more than 10 responses and at least one rejection in the total protocol. In the study presented here only protocols with at least 10 responses and no rejections have been used. The range of responses per protocol is from 10 to 71 $(\overline{\mathrm{X}}=23.2)$.

A review of the literature also revealed 10 studies which correlated the RPRS subscores with success in therapy. These studies are 
summarized in Table I. Of the 6 RPRS subscores FM is shown to be the least effective predictor of success in therapy. FL is the most effective subscore for predicting success in a schizophrenic population. The variance of the FL score for a non-schizophrenic population can be expected to be small, consequently no significant correlations will be found for non-schizophrenics. $M$, Sh and $C$ are the subscores most often found to correlate with success and are possibly more effective for non-schizophrenics than schizophrenics. Small m seems to confine itself to a population actually experiencing stress, such as stutterers, prisoners and neurotics. It might be concluded that, when dealing with a diverse population, all RPRS variables except FM are important variables in the prediction of success in therapy. 


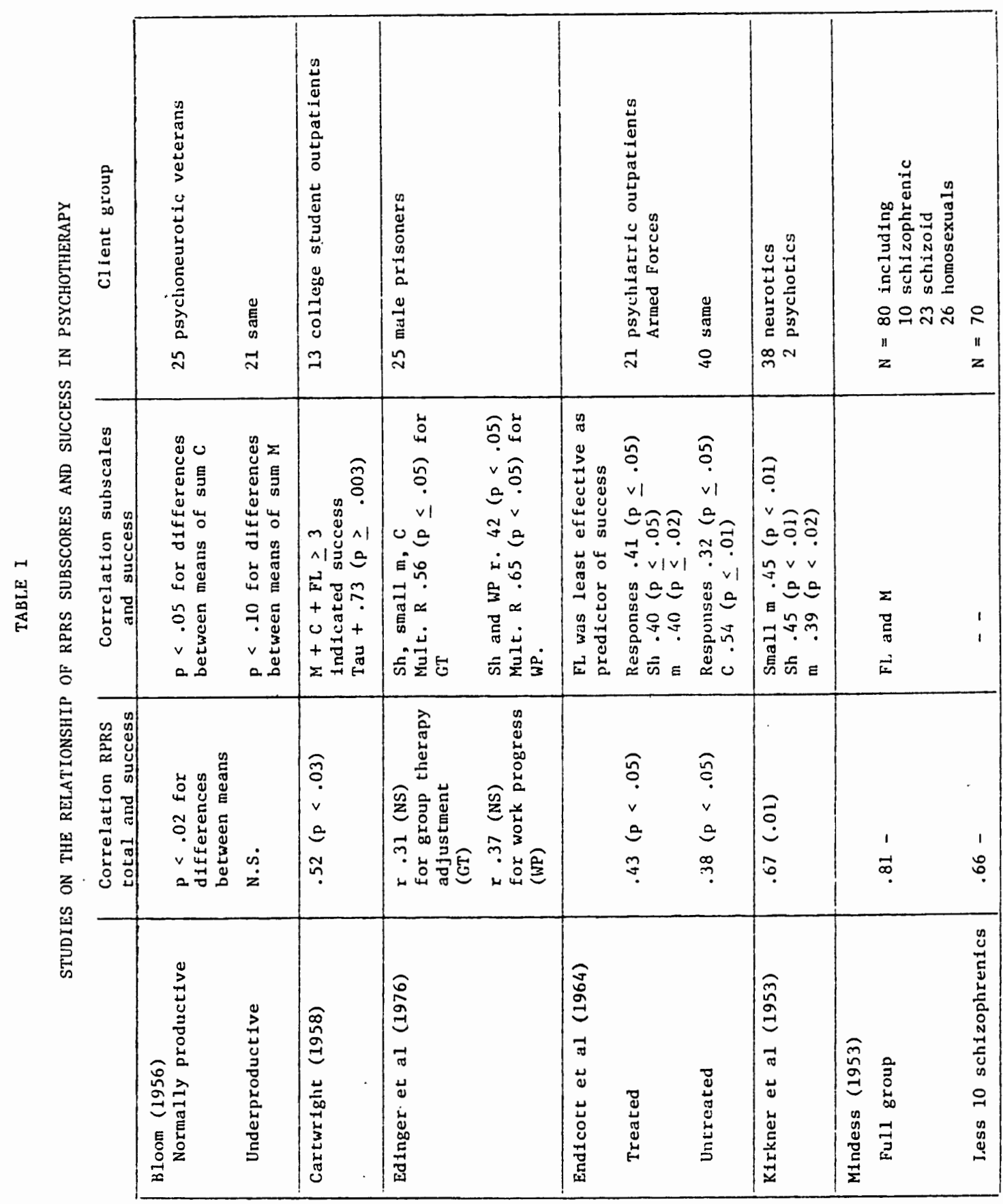




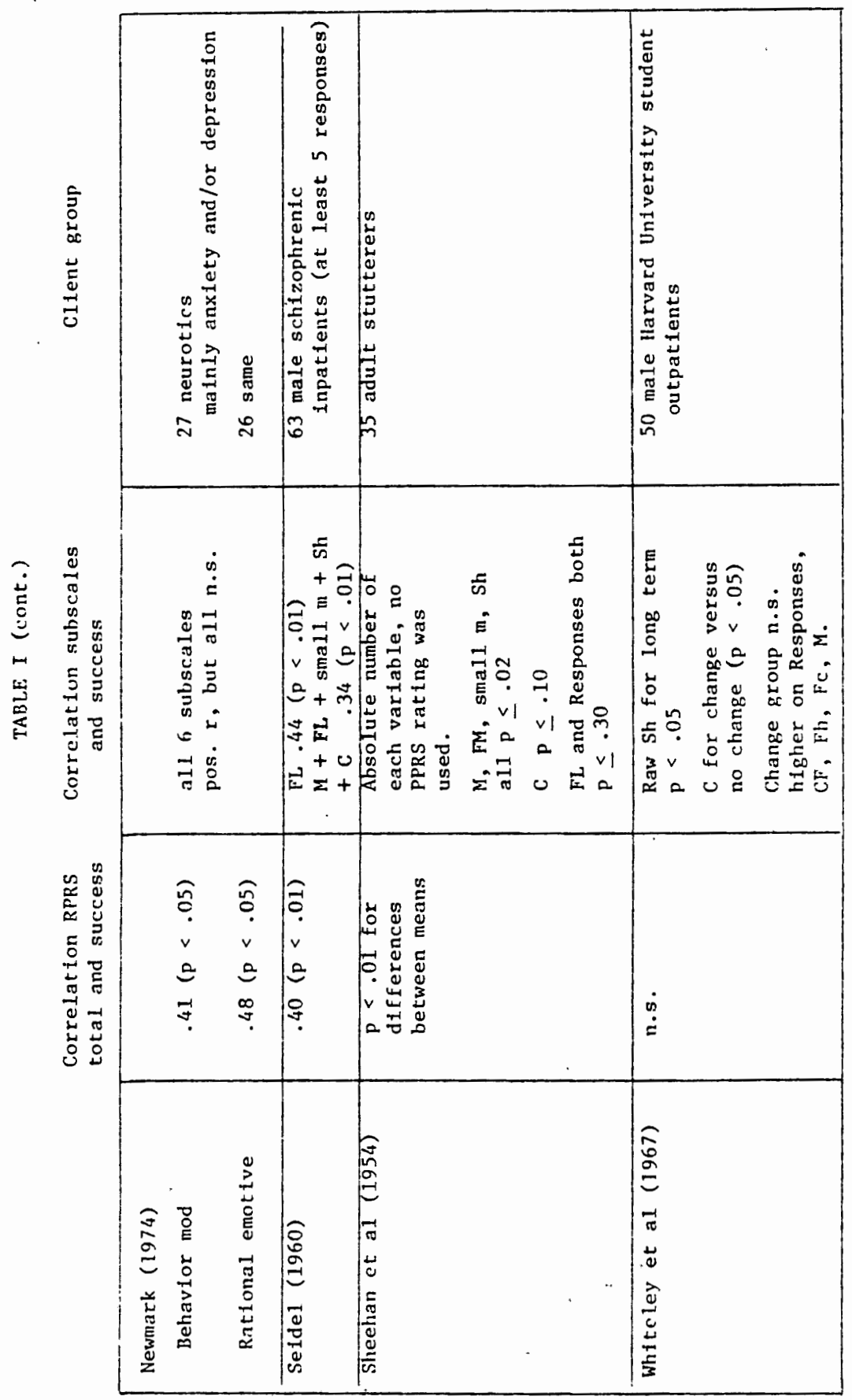


CHAPTER III

\section{INTELLIGENCE AND SUCCESS IN PSYCHOTHERAPY}

\section{A SUMMARY OF THE RESEARCH}

There are two major groups of studies on the relationship between intelligence and success in psychotherapy. The first group deals with continuation in therapy and intelligence. Major studies of this sort are R. A. O'Connell et al, 1972; Salzberg and Bidus, 1966; Garfield and Affleck, 1961; Garfield and Affleck, 1959. These studies state that there is a positive relationship between continuation in therapy and education-intelligence. Garfield and Affleck (1959) state that education after the 8th grade loses its importance as a predictor for attrition. These studies do not, however, have direct relevance to this investigation since they do not deal with intelligence as an intrinsic, causive factor in success of psychotherapy. and will not be discussed further here.

The second major group of studies on psychotherapy and intelligence do deal with the intrinsic, causive relationship of intelligence and ability to predicted benefit from therapy. These studies are, therefore, directly related to this proposed investigation. Accordingly, they are reviewed and summarized in the text below and an overview is presented in Table II.

Although the research in this area is plagued with the problem that different measures are used to identify success in therapy and by 


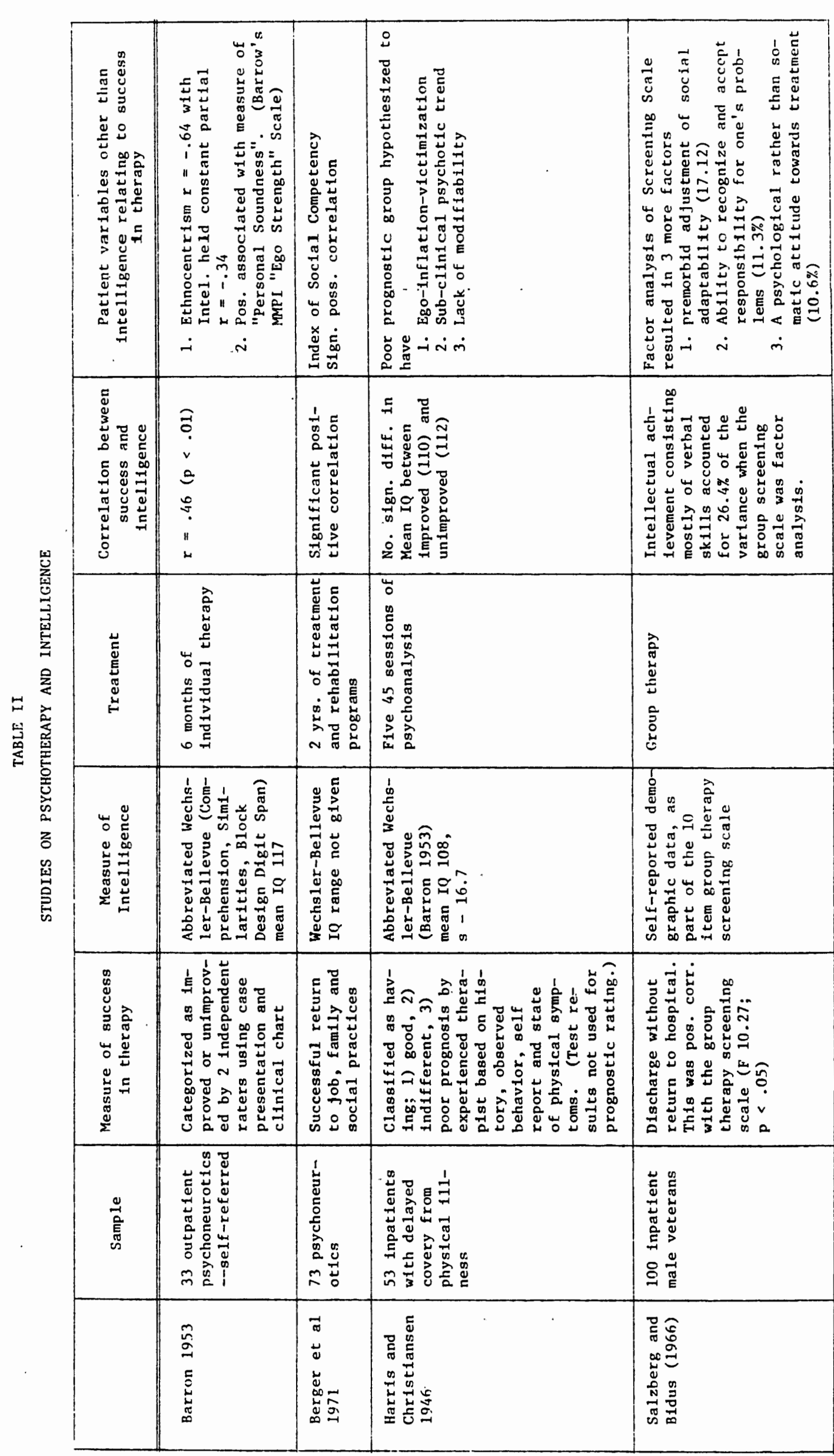


the use of different measures of inteligence, based upon previous research it seems that intelligence has a positive correlation with success in therapy. The question of the nature and degree of the relative contributions of inte11igence and non-intelligence factors to a favorable prognosis for therapy has been examined in this study through a joint factor analytic study of the RPRS and the WAIS. 
CHAPTER IV

IQ AND RPRS - A SUMMARY OF THE RESEARCH

Four studies dealing with IQ and RPRS have been found. They are summarized in Table III. Based upon these studies it seems reasonable to expect that IQ is related to prognosis and that a positive correlation between IQ and favorable prognosis will be found if a sample with a diversity in IQ score is used.

It is interesting to note that Williams et al (1967) found that in the absence of an objective prognostic rating (e.g., the RPRS) the social workers in his study used the parents' report of the child's success in school as a measure of prognosis. The correlation between the social workers' prognostic rating, based on interviews with the parents, and the five psychologists' ratings based on RPRS, was .102 and .285 for the total sample (both non significant).

The Weiss and Edinger (1974) study is the immediate predecessor of the proposed investigation. They accepted the RPRS as a valid measure of the prognosis for success from psychotherapy and found significantly positive overall correlations between the RPRS and the WAIS and especial1y high correlations between RPRS and a combination WAIS verbal subscores. The Weiss and Edinger sample consisted of 15 male hospitalized process schizophrenics and 15 male college students. The correlation 


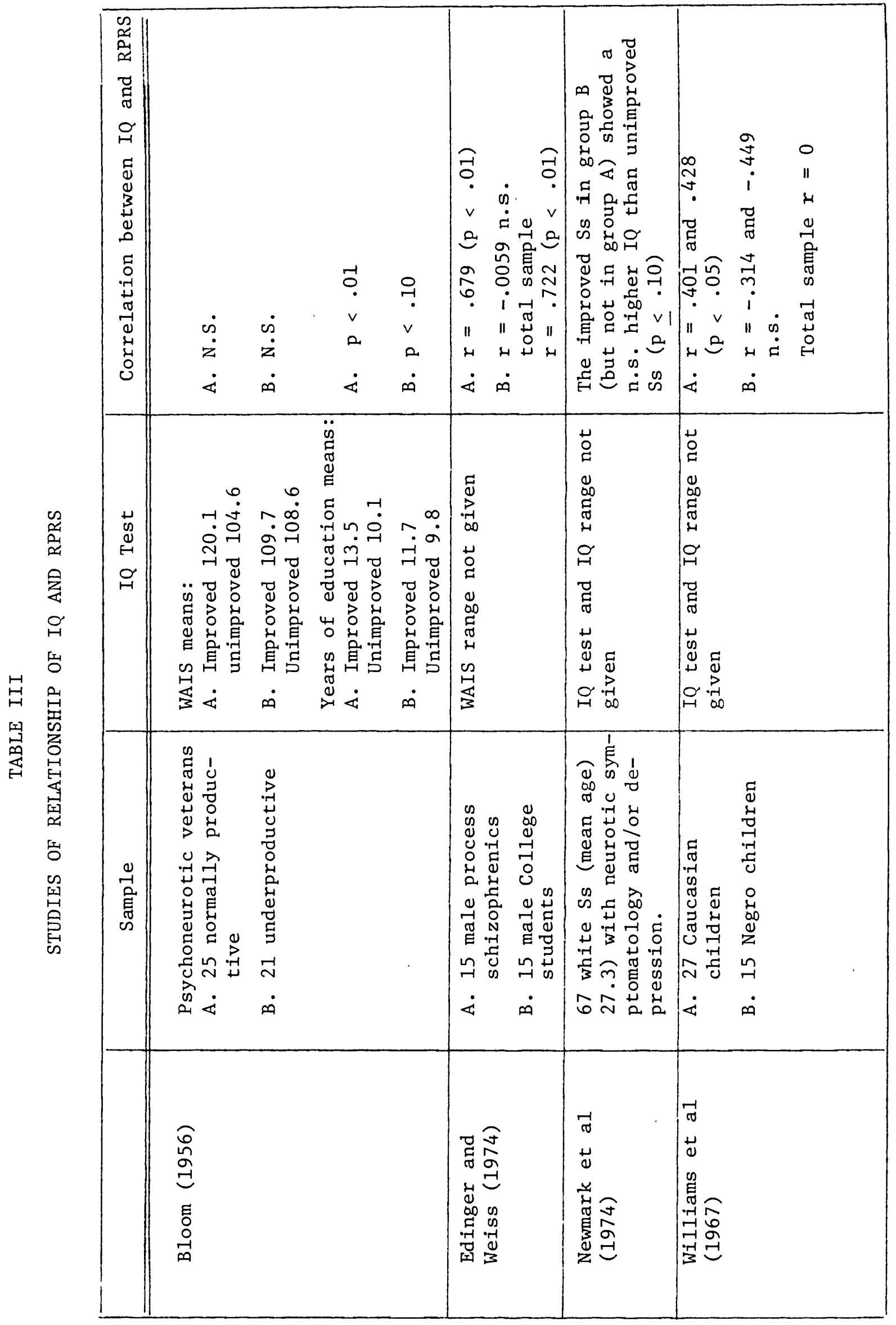


between visual-spatial ability, verbal ability, and RPRS scores was calculated. The visual-spatial ability was measured by the object Assembly and Block Design of the WAIS (OABD). The verbal abilities were measured by the Vocabulary Information and Similarity subtests of the WAIS (VIS). For the entire group, the OABD to RPRS correlation was $.518(\mathrm{p}<.01)$ and the VIS to RPRS correlation was .714 $(\mathrm{p}<.01)$. For the college group above, no significant correlations were found. For the process group the correlation between VIS and RPRS was .630 $(p<.05)$. These results indicate that verbal abilities as measured by the Vocabulary, Information and Similarity subtests are highly correlated with favorable prognosis if the sample used is a heterogeneous one. Weiss and Edinger concluded that the general intelligence factor, "g", might in part be measured by the RPRS and suggested a factor analytic study of the RPRS "in order to understiand its unusual effectiveness as a prognostic indicator in psychotherapy". This study responds to that suggestion. 
CHAPTER V

METHOD

\section{Introduction}

Following the suggestion of Edinger and Weiss (1974) the major analytic technique employed in this study is factor analysis. This technique is applied to groups of variables selected from among the 23 variables listed in Table IV. A precedent for the application of the factor analytic technique to the general problem area of psychotherapy screening and especially to identifying the role of intelligence as a predictor of success in therapy has been provided by Salzberg and Bidus (1966). In their study they analyzed a 10 variable Group Psychotherapy screening scale and found that it included four factors, the first of which was an intellectual achievement factor involving mostly verbal skills.

In addition to factor analysis, descriptive statistics (means and standard deviations) are presented for each of the 23 variables included in the study (Table V). The table of intercorrelations for these variables is also presented and correlations significant at either the .01 or .05 levels are so identified (Table VI). Table $\mathrm{VI}^{\mathrm{A}}$ presents the intercorrelations for the WAIS subscales from the WAIS manual.

In the remainder of this chapter the data analyzed and the factor analytic techniques employed are reviewed. The results of application of the correlations and the factor analysis to the testing of each hypothesis in the study will be discussed in the following chapter. 
TABLE IV

VARIABLES USED IN THIS STUDY

Possible Range

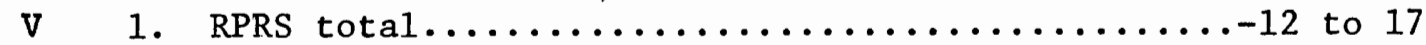

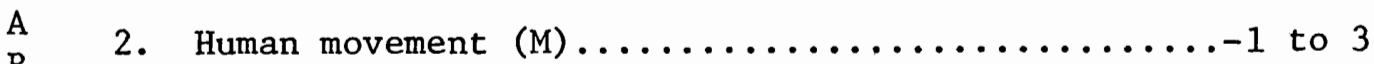

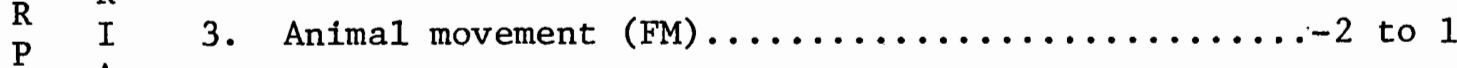

$\mathrm{R} A \mathrm{~A} \quad 4$. Inanimate movement (m) $\ldots \ldots \ldots \ldots \ldots \ldots \ldots \ldots \ldots$ to 2

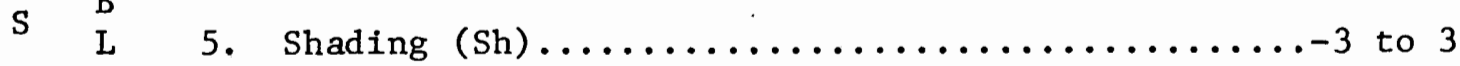

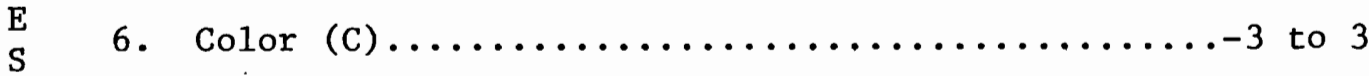

7. Formlevel rating (FL) $\ldots \ldots \ldots \ldots \ldots \ldots \ldots \ldots, 2$ to 5

Mean and Standard Deviation from WAIS Manual

8. WAIS Fu11 Scale Score (FSIQ)............m=100, $s=15$

W 9. WAIS Verbal Score (VIQ) $\ldots \ldots \ldots \ldots \ldots \ldots \ldots \ldots m-100, s=15$

A 10. WAIS Performance Score (PIQ) .............m=100, $s=15$

$S$ 11. Vocabulary, Informator and Similarities (VIS)..m=10, $s=3$

12. Object Assembly and Block Design (OARD)......m=10, $s=3$

A 13. Information (I) $\ldots \ldots \ldots \ldots \ldots \ldots \ldots \ldots \ldots \ldots \ldots \ldots \ldots \ldots \ldots, s=3$

$\mathrm{R}$ 14. Comprehension (Comp) .................... $\mathrm{s}=10$,

A 15. Arithmetic (A) $\ldots \ldots \ldots \ldots \ldots \ldots \ldots \ldots \ldots \ldots \ldots \ldots \ldots \ldots, s=3$

B 16. Similarities $(\mathrm{S}) \ldots \ldots \ldots \ldots \ldots \ldots \ldots \ldots \ldots \ldots \ldots \ldots, \mathrm{m}=10 \ldots \ldots$

$\mathrm{L}$

E

17. Digit $\operatorname{Span}(\mathrm{DS}) \ldots \ldots \ldots \ldots \ldots \ldots \ldots \ldots \ldots \ldots \ldots \ldots \ldots \ldots \ldots, \mathrm{m}=10, \mathrm{~s}=3$

18. Vocabulary (V) $\ldots \ldots \ldots \ldots \ldots \ldots \ldots \ldots \ldots \ldots \ldots \ldots \ldots \ldots \ldots, s=3$

19. Digit Symbol (D sym) $\ldots \ldots \ldots \ldots \ldots \ldots \ldots \ldots \ldots m=10, s=3$

20. Picture Completion (PC) ...............m=10, $s=3$

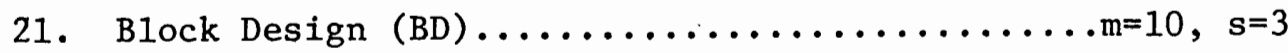

22. Picture Arrangement $(\mathrm{PA}) \ldots \ldots \ldots \ldots \ldots \ldots \ldots \ldots \ldots \ldots \ldots, \mathrm{m}=10, \mathrm{~s}=3$

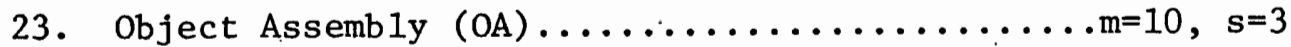

(Age, which was the only socio-demographic variable available, is included in the descriptive and bivariate correlational analyses.) 
TABLE V

\section{MEANS AND STANDARD DEVIATIONS \\ FOR WAIS AND RPRS VARIABLES \\ FROM THE STUDY SAMPLE

$$
\mathrm{N}=52
$$

VARIABLE

AGE

RPRS

M

FM

m

Sh

C

FL

$\mathrm{R}$

FSIQ

VIQ

PIQ

VIS

OABD

I

Comp

A

$\mathrm{S}$

DS

$\mathrm{V}$

D sym

PC

BD

PA

$\mathrm{OA}$
MEAN

25.558

5. 550

1. 385

.173

.865

1. 174

1.169

.784

23. 212

111.000

111.846

108.269

12.312

11.385

11.750

12.442

10.539

12.635

10.962

12. 539

11.077

10.712

11. 558

10.365

11.212
STD. DEV .

8.735

3.991

1.032

.834

.742

1. 811

.863

.474

13.827

15. 732

16.221

17.010

3.035

3. 534

2.943

4. 552

3.190

3. 396

3. 395

3. 489

3.839

2.789

3.691

3.326

3. 892 
కి

$\stackrel{\leftrightarrow}{\lessgtr}$

g

$\stackrel{5}{5}$

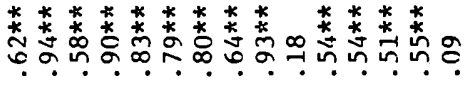

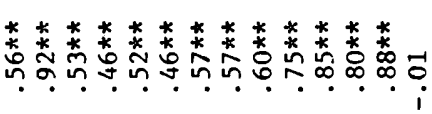

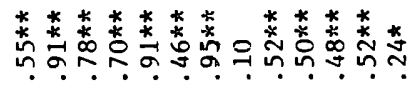

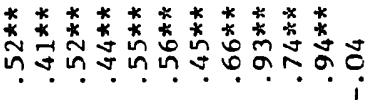

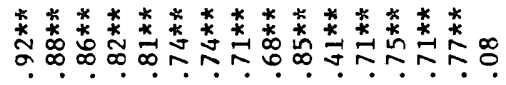

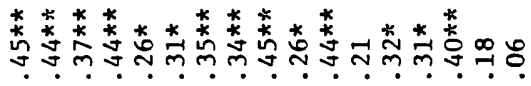

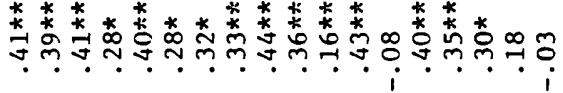

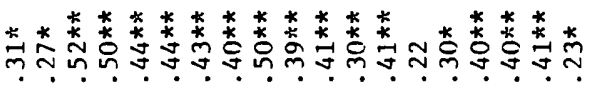

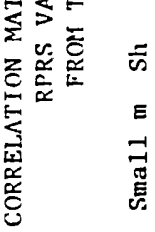

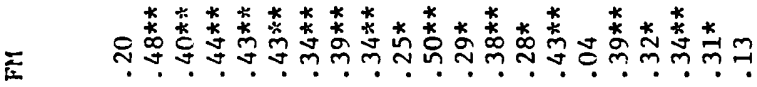

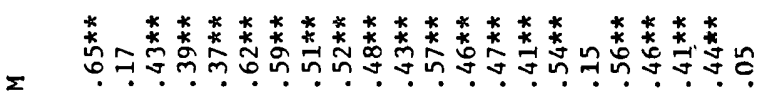

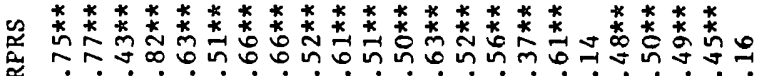

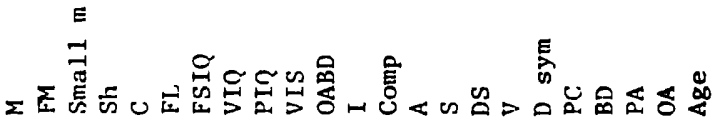

$\delta$

$\stackrel{\sim}{\circ}$

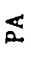

草

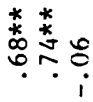

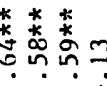

0

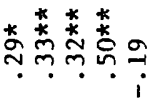

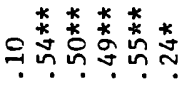

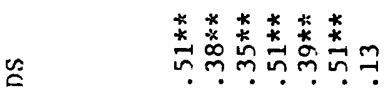

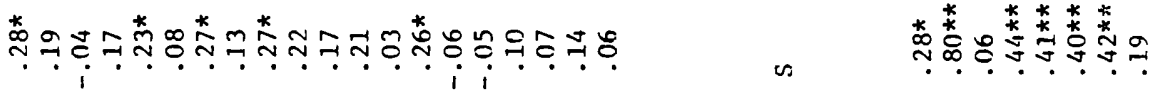

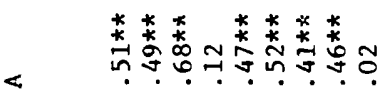

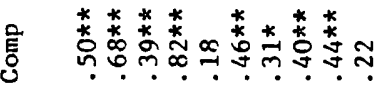

-

命 $\stackrel{0}{\circ}$

a a

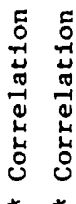

* * 


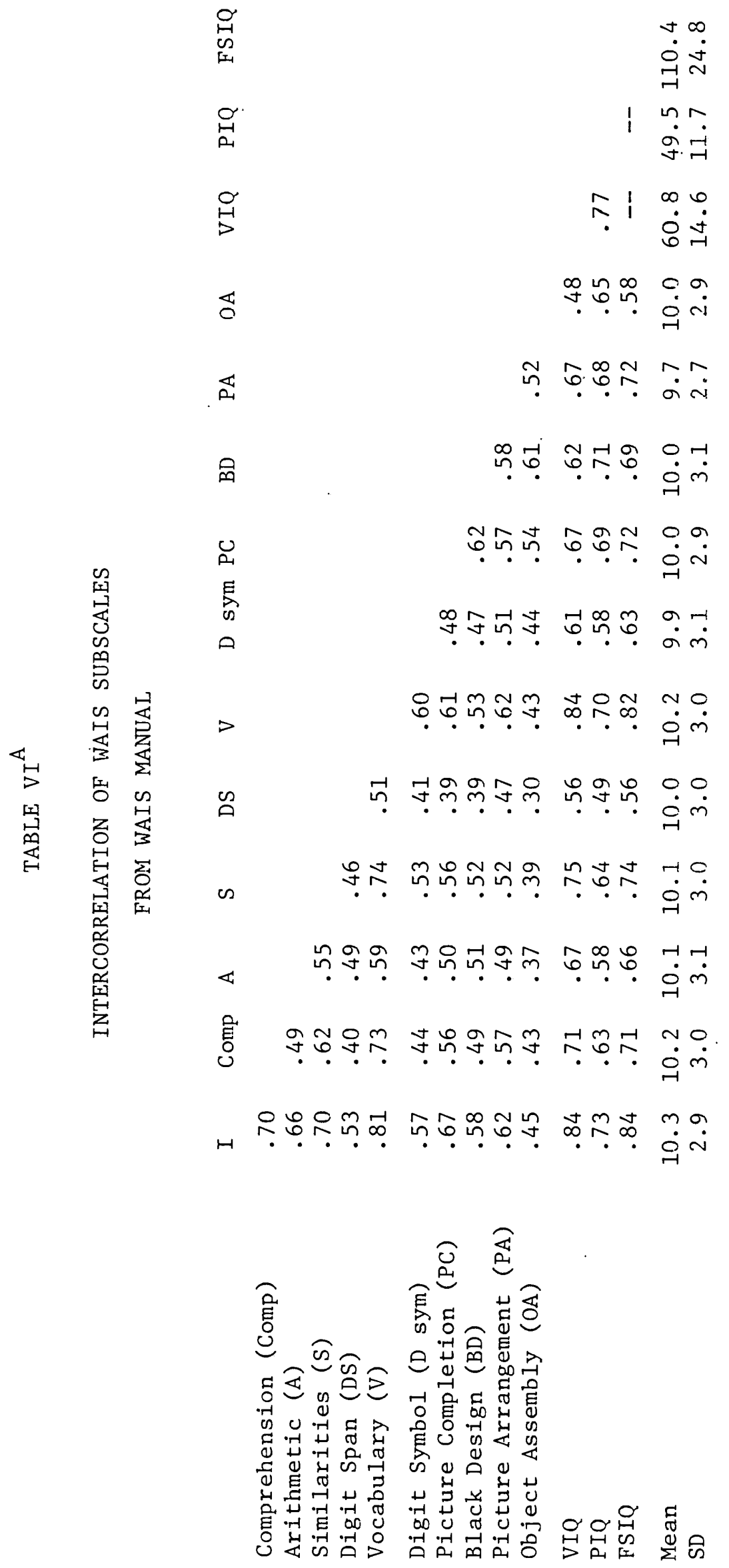


The Sample

The sample selected for this study contains a large variety of subjects to insure maximum variance of all the variables used. Comrey (1973, p. 202) states that "for exploratory work, it is much more important to insure that there is plenty of variance on the factor in the sample than it is to have a representative sample from the population at large."

The sample consisted of 52 subjects, who were either in therapy or applying for therapeutic treatment. Presented problems varied from psychotic reactions to marital difficulties. Twenty-eight clients were identified as in-patients and seventeen as out-patients. Thirty-five of the 52 subjects were female and 17 were male. The range of ages was from 17 to 52 , the mean age was $25.6(s=8.7)$. Thirty-six of the subjects came from the Portland Metropolitan area, specifically from the Cedar Hills Psychiatric Center and Clinic (19), the Portland State University Psychology Clinic (12), the Dammasch State Hospital (3), and the Vancouver Veterans Hospital (2). Eighteen of the subjects were drawn at random from a file of 2000 subjects at the Rorschach Workshops, Bayville, New York. Both the Rorschach and the WAIS were administered to each client within 2 weeks of each other for 49 of the clients. For 2 of the clients the tests were separated by 6 months, because of an interruption in therapy, and for 1 client the tests were separated by 2 months. In all these 3 cases the Rorschach preceded the WAIS.

\section{Data Collection}

A11 subjects had a Rorschach protocol of at least 10 responses with 
no rejections (Bloom 1956). Rejections were only allowed if the protocol had more than 10 responses. The mean number of responses was 23.2 ( $s=13.8)$. Each Rorschach protocol was scored and each scored response rated by two independent raters according to the instructions for the RPRS (Klopfer 1954). The interrater reliability, as measured by the Pearson $r$ between the 2 raters total RPRS score for all 52 subjects, was .95 . When there was a difference in the rating for one of the 6 subscores ( $M, F M, m, S h, C$ and FL) the average of the raw scores was taken. These raw scores were converted to weighted scores and added to arrive at the total RPRS score. Only full WAIS protocols were used so that FSIQ, VIQ and PIQ could be calculated for all the subjects.

Analytic Procedures

Standard bivariate correlational techniques and tests of significance were applied to the data to identify significant relationships between pairs of the 23 variables. Moreover, factor analytic techniques were employed to determine whether selected subsets of the varlables in the study could be reduced to a smaller number of common factors and thereby to determine the basic dimensions or relationships among these variables and the aggregate variables constructed from them, especially the relationships of RPRS to intelligence.

The number of factors to be rotated was arrived at by following Cattell's (1966, p. 297) and Harman's (1960, p. 85) suggestion of using a combination of Kaiser's criterion ("rotate as many factors as there are eigenvalues greater than or equal to unity"). Factor analyses were 
performed using both unity (principal component analyses) and squared multiple correlations (principal factor analyses) estimates of the communalities. The factor analytic results are reported mainly in terms of the principal factor model since it best reproduces the observed correlations rather than merely extracting the maximum variance as does the principal components model. The decision to favor the principal factor results is also in accord with Cattell's rejection of the principal components model for general scientific research because of the unlikelihood of any "real" variables containing in themselves all sources of their variation as that model assumes. This is certainly unlikely with either of the two main sets of variables with which this study deals or with any of the subsets of them. No differences are expected to occur between the principal component and principal factor analysis. Varimax rotation after Kaiser normalization was employed, as a variety of authors suggest, in order to approach simple structure.

Hypotheses

Hypothesis I. (Degree of relatedness of the overall RPRS and WAIS measures): The degree of relationship between performance on the RPRS and the WAIS is moderate and positive.

This hypothesis about the overall relatedness of the RPRS and intelligence will be tested by examining for significance the correlation between the two variables RPRS total and WAIS total.

Hypothesis II. (Degree of relatedness of the overall RPRS and the WAIS verbal and performance subscores). The correlation between the RPRS total and the WAIS Verbal IQ is positive and higher than the 
correlation between RPRS and the WAIS Performance IO.

This hypothesis will be tested by examining for significance and relative magnitude, the correlations between the RPRS total and the WAIS Verbal IQ and between the RPRS total and the WAIS Performance IQ. A t-test will be used to determine whether the difference between these two correlations is significant.

Hypothesis III. (Replication of the Weiss and Edinger, 1974, study). The correlation between the WAIS Vocabulary, Information and Similarities sub-tests combined (VIS) and the RPRS total is positive, significant and greater than the correlation between the WAIS Object Assembly and Block Design sub-tests combined (OABD) and the RPRS total (which is also, however, positive and significant).

This hypothesis will be tested by examining for significance and relative magnitude the correlations between the VIS and the RPRS and between the OABD and the RPRS. A t-test will be used to determine whether the difference between these two correlations is significant.

Hypothesis IV. (The role of intelligence in the RPRS). The first factor in the RPRS and the one making the major contribution to the total variance measured by that scale is a general intelligence factor.

The analysis here consisted of two principal factor analyses with varimax rotation. The first analysis was of the six RPRS subscores. Its results will be examined to determine the factor structure of the scale and the percentages of common variance attributable to each factor. The second analysis was the same as the first except with 
the inclusion of the WAIS total score as a marker variable in order to identify which of the factors in the RPRS factor structure is most closely related to general intelligence.

A third factor analysis was conducted on the six RPRS subscores along with the WAIS verbal and performance subscores now serving in the role of marker variables to determine the nature of the relations between the RPRS subscales and verbal and performance intelligence.

Hypothesis V. (Identification of the nonintelligence factors in the RPRS. There are nonintelligence as well as intelligence factors measured by the RPRS.

To test this hypothesis the results of the same three factor analyses described above will be examined to determine whether or not there are factors in the RPRS factor structure significantly related to intelligence and which RPRS subscales define them.

Two final exploratory factor analyses were conducted using, first, all 23 variables of Table IV, and second, only the six RPRS subscores and the eleven WAIS subscores. The results of these analyses will be used to identify possible future directions for research.

\section{Significance}

It is hoped that the results of this study will add to specific understanding of the reasons for the utility of the RPRS as a predictor of success in psychotherapy and lead to further research for understanding the conditions which contribute to the success of therapeutic interventions. A model of this larger framework is provided in Figure 1. 


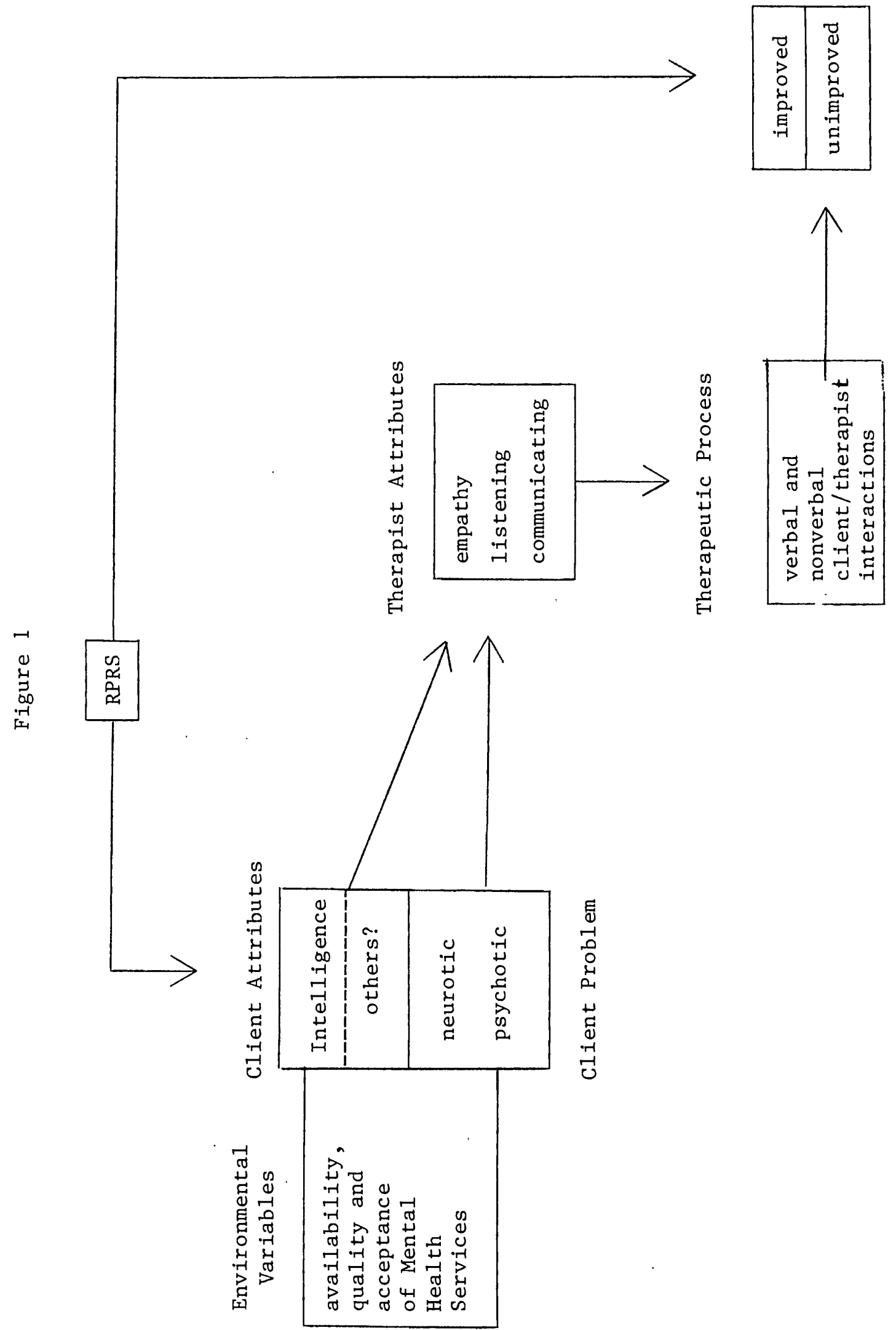


This model shows that client variables, environmental variables and client problems are important components in a client's decision to seek therapy. The client attributes and problems interact with the therapist's attributes which together, through the therapeutic process, result in an outcome of therapy. This outcome can be defined as improved or unimproved. The RPRS attempts to measure client variables which have an effect on the outcome of therapy .

This thesis attempts to identify and assess the impact of those client variables measured by the RPRS. Greater understanding of these variables and their effects on the outcome of therapy can have implications for the prediction of the success of therapy, the selection of clients, theoretical understanding of the therapeutic process and the design of more effective therapeutic treatments. 


\section{CHAPTER VI}

\section{RESULTS}

This chapter presents the results of the quantitative analyses performed. The forms of analysis as mentioned before, include descriptive statistics, correlations and factor analysis. The quantitative results of these analyses for the appropriate variables will be presented along with such technical explanation and immediate interpretation as is necessary for a clear reading of the results. In depth interpretation of the results is reserved for the following chapter.

\section{Descriptive Statistics}

Means and standard deviations for each of the 23 variables and for age as well are given in Table V. This sample has a somewhat higher FSIQ than one would expect to find in a random sample of the general population, namely 111 versus 100. The mean RPRS score of 5.55 with a standard deviation of 3.00 shows that prognostic groups I through IV (Klopfer 1954) are represented in this sample. Of the 52 subjects, $68 \%$ fall in groups II and III. This is the distribution found in other studies dealing with the RPRS (Garwood 1978). Prognostic group $\mathrm{V}$ and VI which Klopfer describes as "poor treatment prospect" and "hopeless case" respectively, have rarely been found in previous research. In a review of 11 different studies using a total of 481 clients only 1 client was mentioned to belong to prognostic group $\mathrm{V}$ and 4 to group VI. No restrictions on the number of responses in the 
Rorschach was noted for the 2 studies reporting these clients (Filmer-Bennett, 1955; Johnson, 1953). It is unlikely that a valid Rorschach protocol (one with at least 10 responses and no rejections) can be obtained from such cases. The subjects in this sample were also required to produce a complete WAIS protocol so that FSIQ, VIQ and PIQ could be calculated.

\section{Correlations}

The purpose of this subsection is to evaluate the appropriateness of these data for use in later factor analysis. Specifically, the intercorrelations of the WAIS, RPRS and age variables will be examined in light of correlations found in past research.

The intercorrelations for the 23 variables plus the age variable are given in Table VI. The WAIS manual gives a table of intercorrelations of the WAIS scales which is presented in Table VI-A. The table of correlations for the 25-34 age group has been used, since it best represents the present sample. The correlations found in this study are congruent with the ones given in the WAIS manual, except for the D sym sub-test. The correlations of the $D$ sym with the verbal subtests are consistently lower here. D sym involves the ability to master a new and essentially alien task within a brief time span. Through middle age, this test is one of the poorest measures of general intelligence $(G)$. The largest discrepancy is found between $D$ sym and Similarities (.06 for this study versus .53 from WAIS manual). Similarities ranks as a good measure of $G$. This seems to indicate that the present sample varies from the WAIS manual sample in the specific abilities measured by D sym. Zimmerman and Woo-Sam (1973) state that the 
particular aspects of the intelligence evaluated by the D sym seems to vary across age group and diagnostic group. Saunders (undated) defines $D$ sym as a measure of "psychometabolic rate" influenced by such variables as drugs, brain damage and psychological state. Wechsler (1958, pp. 171-172) states that in schizophrenia and anxiety state reaction the expectation is that scores should deviate 1.5 to 2.5 units below the mean sub-test scores. The results of the present study confirm that the D sym sub-test is sensitive to the psychological state of the person. In contrast to the sample used in the WAIS manual, the present sample consisted of persons in need of therapeutic assistance. Some of the subjects were on prescribed drugs when the test was taken.

The age variable has been included in the correlation matrix and is shown to be slightly negatively correlated with the WAIS performance subscales and with $C$. Although none of these correlations are significant it does tend to confirm the validity of the data used. Age is shown to be positively correlated with shading ( $\mathrm{Sh})(\mathrm{r}=.23 \mathrm{p} \leq .05)$. In their review of the research on State and Trait anxiety, Auerbach and Spielberger (1972) state that the shading variable is the best Rorschach indicator of state anxiety. Shading, namely Klopfer's FK score, is also assumed to be related to introspection (Klopfer et al 1954). No empirical studies dealing with this subject have been found.

The correlation between $M$ and FSIQ needs special mention because of the previous research done on the relationship between human movement (M) and intelligence. High $M$ has been found to indicate above 
normal intelligence (Auerbach and Spielberger 1972) and is positively correlated with independent measures of intelligence (Hirt 1972). In the present study $M$ was found to correlate higher with FSIQ than any of the other RPRS subscales. The correlation of .616 $(\mathrm{p}<.001)$ indicates that approximately $38 \%$ of the variance in $M$ can be accounted for by FSIQ.

\section{Factor Analysis}

The results of the varimax rotated, principal factor analyses on five different sets of variables are presented in Tables VII, VIII, IX, $\mathrm{X}$, and XI. Principal component (P.C.) analyses were also done to allow for comparisons with the results found in the principal factor. (P.F.) analyses. The factor loadings of the principal factor analysis were generally somewhat lower than the factor loadings of the P.C. analysis. This is explained by the fact that in P.C. analysis all variance, including specific and error variance is accounted for as common variance. In principal factor analysis specific and error variance are excluded from the common variance. The principal component in general confirmed the results found in P.F. analysis, except in the last factor analysis (RPRS subscores and WAIS subscales). These difference will be discussed in the following chapter.

Only variables with a factor loading of .50 or larger are used to interpret the results of the factor analysis. This is recommended by Comrey (1973, p. 200) when the sample is small. A sample of 52 has a low reliability of the correlation coefficients. To compensate for this the higher factors loading of $\geq .50$ will be used to interpret the results. 


\section{TABLE VII}

\section{FACTOR MATRIX OF THE 6 RPRS SUBSCALES}

(Varimax Rotation)

$$
\mathrm{N}=52
$$

FACTOR 1

FACTOR 2

M

.6463

.3622

FM

$.7044 \quad .3850$

m

.0310

.5211

$\mathrm{Sh}$

C

.4257

.4734

.5221

.2050

FL

.7093

$-.1189$ 
TABLE VIII

FACTOR MATRIX OF THE 6 RPRS SUBSCALES

(Varimax Rotation)

$$
\mathrm{N}=52
$$

$\begin{array}{rr}\text { FACTOR 1 } & \text { FACTOR 2 } \\ .5670 & .5170 \\ .5677 & .4646 \\ -.0016 & .4373 \\ .3650 & .5671 \\ .4827 & .2718 \\ .8212 & -.0851 \\ .5787 & .4492\end{array}$


TABLE IX

FACTOR MATRIX OF THE

6 RPRS SUBSCALES, VIQ AND PIQ

(Varimax Rotation)

$\mathrm{N}=52$

FACTOR 1

FACTOR 2

M

.6806

.3462

FM

.6266

.2999

m

.0516

.4986

Sh

.4727

.4903

C

.5127

.1748

FL

.7200

.1632

VIQ

.6852

.3696

PIQ

.5965

.2393 
TABLE $X$

FACTOR MATRIX OF ALL 23 VARIABLES

(Varimax Rotation)

$$
\mathrm{N}=52
$$

FACTOR 1 FACTOR 2

FACTOR 3

.3149

.3214

.1507

.1787

.2314

.2671

.2931

FL

FSIQ

VIQ

PIQ

VIS

OABD

I

Comp

A

$\mathrm{S}$

DS

$\mathrm{V}$

D sym

PC

BD

PA

$\mathrm{OA}$
.2503

.3033

.1430

$-.0352$

.2809

.0755

.1432

.6524

.3136

.9217

.2316

.9044

.2638

.1783

.3144

.1513

.4849

.2655

.5789

.5689

.7858

.6681

.8532
.8729

.6195

.7816

.1558

.5252

.5530

.5379

.3066

.3117

.2376

.2693

.1890

.1049

.4003

.2364

.3229

.1275

.3083

$-.0095$

.3539

.2461

.3026

.1186
FACTOR 4

.3351

.0747

.0789

.7912

.3320

.0151

$-.3107$

.0247

.0682

$-.0169$

.0805

.1139

.1380

.0959

.0518

.0345

$-.0115$

.0684

$-.0581$

$-.1642$

.0339

$-.0330$

.1612 
TABLE XI

FACTOR MATRIX OF THE 6 RPRS SUBSCALES

AND 11 WAIS SUBSCALES

(Varimax Rotation)

$$
\mathrm{N}=52
$$

FACTOR 1

M

FM

$\mathrm{m}$

Sh

C

FL

I

Comp

A

$\mathrm{S}$

DS

$\mathrm{V}$

D sym

PC

BD

PA

OA
FACTOR 2

$$
\begin{array}{r}
.2902 \\
.1190 \\
-.0366 \\
.2869
\end{array}
$$$$
-.0129
$$

.1588

.2839

.2273

.2979

.1648

.5171

.2753

.6211

.5117

.7057

.6207

.8387
FACTOR 3

$$
\begin{array}{r}
.5578 \\
.8080 \\
.1829 \\
.4417
\end{array}
$$$$
.2855
$$$$
.3136
$$$$
-.0048
$$$$
.4657
$$$$
.0611
$$$$
.2681
$$$$
.1189
$$$$
.2526
$$$$
.0614
$$$$
.2157
$$$$
.0925
$$$$
.2021
$$$$
.1613
$$

FACTOR 4

$$
\begin{array}{r}
.3250 \\
.2743 \\
-.0187 \\
.1188
\end{array}
$$

.6079

.3823

.2537

.0852

.4181

.2901

.1134

.3086

$-.1260$

.4848

.4716

.3697

.1185 
CHAPTER VII

CONCLUSIONS

This chapter will be chiefly devoted to applying the results of the data analysis on the five research hypotheses. Some additional ideas suggested by the results but extending beyond the framework established by the hypotheses will be presented at the end of this chapter.

Conclusions about the Hypotheses

Hypothesis I. (Degree of relatedness of the overall RPRS and WAIS measures): the degree of relationship between performance on the RPRS and the WAIS is moderate and positive.

The correlation between the two variables RPRS total and WAIS total is found to be .6575 ( $\mathrm{p}<.001$ ). This confirms the first hypothesis (See Table VI, page 19).

Hypothesis II. (Degree of relatedness of the overall RPRS and the WAIS verbal and performance subscores): the correlation between RPRS total and the WAIS Verbal In is positive and higher than the correlation between RPRS and WAIS Performance IQ.

The correlation between RPRS total and WAIS Verbal IQ was found to be $.6552(\mathrm{p}<.001)$ and between RPRS total and WAIS Peṛformance IQ .5236 $(p<.001)$ (See Table 6). The t-test for differences between these 
two correlations resulted in $t=1.42, \mathrm{df}=49(\mathrm{p}<.10)$. This does not confirm the second hypothesis, since the significance level is only .10 .

Hypothesis III. (Replication of the Weiss and Edinger, 1974 study): the correlation between the WAIS vocabulary, Information and Similarities subtests (VIS) and the RPRS total is positive, significant and greater than the correlation between the WAIS Object Assembly and Block Design subtests combined (OABD) and the RPRS total (which is also, however, positive and significant).

The correlation between VIS and RPRS was found to be .6045 $(\mathrm{p}<.001)$. The t-test for differences between these two correlations resulted in $t=.93, \mathrm{df}=49(\mathrm{p} \geq .10)$, indicating that although the VIS correlation is somewhat higher than the OABD correlation, this difference is not significant at the <.10 level. Therefore, the third hypothesis could not be confirmed.

Weiss and Edịnger (1974) do not report a t-test for differences between the two correlations. The VIS, OABD correlation needed to compute the t-test was not reported either. The question whether the Weiss and Edinger study showed a significant difference remains.

Hypothesis IV. (the role of intelligence in the RPRS): the first factor in the RPRS and the one making the major contribution to the total variance measured by that scale is an intelligence factor.

Hypothesis V. (identification of the non-intelligence factors in the RPRS): there are non-intelligence as well as intelligence factors measured by the RPRS. 
To test these 2 hypotheses Principal Factor analyses were conducted. The first was on the 6 RPRS subscores alone; the second was on the 6 RPRS subscores with the WAIS total score (FSIQ); and the third was on the RPRS subscores with the two WAIS subscores (See Tábles VII, VIII \& IX, pages $32,33 \& 34)$. Factor analysis of the 6 RPRS subscores alone resulted in the identification of 2 factors. Of the 6 RPRS variables, four group solidly together on the first factor which accounts for $83.6 \%$ of the extracted common variance (Table VII). Those 4 variables are in order FL, $\mathrm{M}$ and $\mathrm{C}$. Only small $\mathrm{m}$ is obviously a determinant of the second factor which accounts for $6.4 \%$ of the extracted common variance. Sh straddles the two factors almost equally. Moreover, its lack of any factor loading above .5 adds to its ambiguity.

The PF factor analysis of the 6 RPRS variables with the inclusion of FSIQ also resulted in 2 factors (Table VIII). The results of this analysis show that the first RPRS factor is indeed an intelligence factor. The order of the 4 RPRS variables assigned to intelligence in terms of the strength and clarity of their relationship to intelligence is accordingly $\mathrm{FL}, \mathrm{FM}, \mathrm{M}$ and $\mathrm{C}$. The second non-intellective factor is primarily defined by small $\mathrm{m}$. In this second analysis $\mathrm{Sh}$ revealed itself by a factor loading above .5 on the second factor as more obviously predominantly also non-intellective. FM and $M$ although still primarily aligned with the first intellective factor, were confirmed as "straddlers" between the first and second factors. Since $\mathrm{C}$ in this analysis does not have factor loadings above .5 on either factor in this analysis $(.48, .27)$ and barely did so in the previous analysis 
$(.52, .20)$, its alignment is ambiguous.

Finally, the Principal Factor analysis of the RPRS subscales and the two WAIS subscales VIQ and PIQ resulted again in 2 factors. The first factor remains an intelligence factor with VIQ and PIQ almost equally aligned with it. Of the 6 RPRS variables, FL again emerges as the most intellective of the RPRS variables, followed, in order, again by $M$ and $F M$. The remaining second factor is a non-intellective factor which is chiefly defined by small $\mathrm{m}$. The Sh variable is confirmed to have substantive non-intellective qualities, and C remains shrouded in mystery.

From the three factor analyses the following conclusions may be drawn concerning the final two hypotheses:

1. The first factor of the RPRS is an intelligence factor (confirmation of Hypotheses IV). This factor accounts for approximately $85 \%$ of the common variance. The most intellective RPRS variable is FL, followed by FM and M in that order.

2. There is a non-intelligence factor in the RPRS (confirmation of Hypotheses V). Small $\mathrm{m}$ is the most obviously nonintellective RPRS variable. Sh is the only other RPRS variable possibly possessing a substantive, non-intellective dimension. FM and $M$ do, however, possess some non-intellective aspects.

3. The status of $\mathrm{C}$ with respect to intelligence is ambiguous.

Conclusions about the Exploratory. Factor Analyses

Two final factor analyses were conducted to explore possible future directions for research. The first factor analysis involved all 23 variables of Table IV. Principal factor analysis resulted in 4 independent factors after varimax rotation (See Table $\mathrm{X}$ ). The first factor is a 
verbal intelligence factor and accounts for $71.5 \%$ of the common variance. Of the 16 WAIS variables, the Information (I) subscale is the variable which best defines this factor, followed in order by VIQ. None of the 7 RPRS variables have a factor loading of $\geq .29$. These are M, RPRS, FL. The second factor is a performance intelligence factor and accounts for $14.0 \%$ of the common variance. The most important determinants of this factor are in order: PIQ, OABD, OA, $\mathrm{BD}, \mathrm{PA}$. Of the 16 WAIS variables, D sym seems to be the variable most determined by this factor. In absolute value of factor loadings this variable only ranks number 7 on this factor, but the absolute value of the factor loadings on the other 3 factors is extremely low for this variable. None of the 7 RPRS variables have factor loadings of $\geq .50$ on this factor. The RPRS variables loading $\geq .28$ are $M(.30)$ and $S h(.28)$. Factor 3 is the RPRS factor and accounts for $9.1 \%$ of the common variance. FM seems to be the most important determinant of this factor followed by RPRS, M, C, FL and Sh. None of the WAIS variables have a factor loading $\geq .50$ on this factor. The WAIS variables loading $\geq .35$ are Comp (.40) and PC (.35). Factor 4 is, like the last factors in the previous analyses, mainly determined by small m. It shows itself clearly to be a non-intellective factor. The conclusions from this analysis can only be tentative, since factor analysis of a large number of variables requires a larger sample than used in this study. The conclusions are:

1. Small $\mathrm{m}$ separates itself from the other 5 RPRS subscales as an independent non-intellective variable. Sh is the only other RPRS subscore with a substantive non-intellective dimension; thus, confirming the results of the previous factor analysis. 
2. The WAIS variable $I$ is the most factorially pure contribution to the verbal intelligence factor.

3. $M$ and FL and maybe $C$ and $S h$ are to some small degree contributors to the verbal intelligence factor.

4. The WAIS subscales which are important contributors to the performance intelligence factor are $\mathrm{OA}, \mathrm{BD}$ and $D$ sym. Of these variables $D$ sym is the most factorially pure variable on this factor.

5. $M$ and $S h$ are to some small degree contributors to the performance intelligence factor.

6. FM is the main determinant of the RPRS factor and the most factorially pure RPRS variable after small $\mathrm{m}$. The other contributors to the RPRS factor are in order: RPRS, $\mathrm{M}, \mathrm{C}, \mathrm{FL}, \mathrm{Sh}$.

7. The WAIS variables which contribute most to the RPRS factor are Comp and PC.

These conclusions are supported by the PC factor analysis.

The second exploratory factor analysis involving the 6 RPRS subscales and the 11 WAIS subscales resulted in 4 independent factors after varimax rotation (See Table XI). The first factor is a verbal intelligence factor accounting for $71.2 \%$ of the common variance. Of the 11 WAIS subscales the Information subscale again emerges as the variable which best describes this factor, followed in order by $\mathrm{V}, \mathrm{S}$ and Comp. None of the 6 RPRS subscales have loadings $\geq .50$ on this factor. Three of the RPRS subscales have loadings $\geq .50$ on this factor. Three of the RPRS subscales have loadings $\geq .29$, namely small $\mathrm{m}$, Sh and M. The second factor is a performance intelligence factor and accounts for $13.7 \%$ of the common variance. Again D sym seems to be the WAIS variable which is best described by this factor: it ranks number 3 as the variable with the highest factor loading, but the correlations of this variable 
with the other factors is extremely low; the other WAIS variables which are important contributors to this factor are $\mathrm{OA}, \mathrm{BD}, \mathrm{PA}$ and DS. None of the 6 RPRS subscores have loadings $\geq .50$ on this factor. Two of the RPRS variables have loadings of $\geq .29$; namely Sh and M. The third factor is the RPRS factor and accounts for $9.7 \%$ of the common variance. The main determinant of this factor is FM, followed In order by $M$, Sh, the WAIS subscale Comp, FL and $C$. In the previous analyses Comp shows itself as the most important WAIS var1able on the RPRS factor. PC was indicated as the second most important WAIS variable on this factor. In this analysis PC does not reveal itself on the third factor but is straddled between the second and fourth factor. The fourth factor is, in contrast with the prevlous anlaysis, somewhat determined by intelligence and performance intelligence in particular. This factor accounts for $5.4 \%$ of the common variance. The most important determinant of this factor is the RPRS variable $C$, followed in order by the WAIS variable PC which is straddled between factors 2 and 4 , A which is straddled between factor 1 and 4 , the RPRS variable FL which straddles factors 3 and $4, B D, P A$, the RPRS variables $M$ and FM. It might be noted that small $\mathrm{m}$ is conspiciously absent on this last factor. This is in contrast to the previous analyses and to the principal component analysis of the 6 RPRS and 11 WAIS variables. In the principal component factor analysis smal1 $\mathrm{m}$ has a loading of .84 , followed by $\mathrm{Sh} .54$ and FM..32, and is clearly a non-intelligence factor. FM, C; FL and M all load solidly on the third factor in principal component factor analysis $(.79, .67$, 
$.66, .64$, respectively), while Sh straddles factor 2 and $3\left(\mathrm{~F}_{1} .33\right.$, $\mathrm{F}_{2}$.41). In the principal component factor analysis the commonality of sma11 m was .75 and in principal factor analysis .11. This indicates that the variance in small $\mathrm{m}$ is largely specific variance, which was extracted as common variance in the principal component factor analysis. C, FL and Sh also had lower communalities in principal factor analysis when compared with the principal component factor analysis, although the difference is not as dramatic as for smal1 $\mathrm{m}$ (C .71 and .40 , FL .58 and .31, Sh .60 and .38).

The tentative conclusions from the principal factor analysis of the 6 RPRS and 11 WAIS variables are:

1. Small $m$ does not reveal itself as the main determinant of an independent factor but appears to have a large amount of specific variance, which is non-intellectual.

2. The first factor. is a verbal intelligence factor. The WAIS variable $I$ is the most important determinant of this factor.

3. The 3 RPRS variables, which to some small degree contribute to this verbal intelligence factor, are. small $\mathrm{m}$, Sh and $M$.

4. The second factor is a performance intelligence factor. D sym and $\mathrm{OA}$ seem to be the most important determinants of this factor, followed by $\mathrm{BD}$.

5. The RPRS variables which to some small degree contribute to this performance intelligence factor are Sh and $M$.

6. The third factor is a RPRS factor. The main determinant of this factor is FM, followed by' $M$ and Sh, Comp, FL and $C$ in that order.

7. Comp is the most important WAIS variable contributing to the RPRS factor. 
8. The fourth factor has the RPRS variable $\mathrm{C}$ as the most important contributor, followed by PC, A, FL, $\mathrm{BD}, \mathrm{PA}, \mathrm{M}$ and FM.

9. The most important WAIS variables on the fourth factor are PC (straddled between 2 and 4) and A (straddled between 1 and 4 ).

In interpreting the results of these factor analyses it has been taken into account that the range of the RPRS variables is considerably lower than the range of the WAIS variables. Because the range of the RPRS variables is smaller, lower correlations can be expected for these variables. Consequently, the distribution of the RPRS variables over the 4 factors has been emphasized. Summarizing the 2 exploratory factor analyses we find:

1. Small $\mathrm{m}$ is non-intellectual and separates itself from the other 5 RPRS variables. Small $\mathrm{m}$ is generally regarded as an indicator of subjective discomfort.

2. M, FL and Sh seem to possess some verbal intellectual dimensions compatible with the verbal dimension measured by $I$. The ability to draw upon and verbalize past experiences seems to best describe this dimension.

3. $M$ and $S h$ also seem to possess some performance intellectual dimension compatible with the performance intelligence measured by $\mathrm{OA}, \mathrm{BD}$ and $\mathrm{D}$ sym. Ability to concentrate and to integrate seems to best describe this dimension.

4. The most important determinant of the RPRS factor is FM, followed by M, Sh, FL, C, while Comp is the most important WAIS variable contributing to this factor. This factor seems to be best described by the ability to control impulses.

5. Another dimension of the RPRS is found only in the PF analysis of the 6 RPRS and 11 WAIS variables and is the most tentative one. $\mathrm{C}$ is the most important determinant of this factor, followed by PC, A, FL, $B D, P A, M$ and FM. The ability to be aware of and the ability to respond to demands of the environment seems to best describe this factor. 
CHAPTER VIII

SUMMARY AND RECOMMENDATIONS FOR FUTURE RESEARCH

Summary

In this study bivariate and multivariate (factor analysis) correlational analyses of the 6 RPRS scales and the WAIS were performed on a sample of 52 subjects with a wide range of variance in their performance.

Major conclusions of the study are:

1. The RPRS and WAIS measures are strongly, positively related with approximately $43 \%$ of the variance in the RPRS total accounted for by the variance in the WAIS total intelligence score.

2. Verbal intelligence is slightly higher related to the RPRS than performance intelligence $(p<.10)$. Approximately $43 \%$ of the variance in the RPRS total can be accounted for by the variance in the verbal intelligence score of the WAIS, and approximately $27 \%$ of the variance in the RPRS total can be accounted for by the variance in the performance inte1ligence score of the WAIS.

3. A non-significant, higher portion (approximately 36\%) of the variance in the RPRS total can be accounted for by the variance in the vocabulary, information and similarities subscales than can be accounted for by the variance in the object assembly and block design subscales together (approximately $26 \%$ ).

4. Factor analysis of the RPRS results in 2 factors, the first factor is an intelligence factor accounting for approximately $85 \%$ of the common variance. FL, FM and $M$ are the determinants of this factor. The second factor is a non-intelligence factor accounting for approximately $15 \%$ of the common variance. Small $m$ is the main determinant of this factor. Sh is the only other variable possessing this non-intellective dimension. This second factor can be best described as an indication of subjective discomfort. $C$ is not clearly intellective or non-intellective. 
5. Exploratory factor analysis indicated the following:

a. M ranks with Sh as the most factorially complex of the RPRS subscales. $M$ is an intellective variable with both verbal and performance intelligence dimensions. It is only surpassed by FL as the strongest intellective variable.

b. FM follows $M$ as the 3 rd most important intellective variable. The intellectual dimension in FM is the one most germane to the RPRS subscales. It has no discernible amount of verbal or performance intelligence.

c. Small $\mathrm{m}$ is the only RPRS variable which does not possess an intellective dimension.

d. Sh is an intellective as well as a non-intellective subscale. It possesses a verbal as well as a performance intelligence dimension.

e. C is, after small $\mathrm{m}$, the least important intellective variable, possibly possessing some degree of verbal intelligence.

f. FL is the most important intellective subscale. The intellective dimension tends to be more of a verbal intelligence dimension.

Directions for future research

The RPRS variables which contribute most to the verbal intelligence factor are $M$ and $F L$ followed by $S h$ and perhaps $C$. The RPRS variables which contribute most to the performance intelligence factor are $\mathrm{M}$ and $\mathrm{Sh}$. This seems to be indicative that, when inte1ligence is known, FM and small $\mathrm{m}$ become the most important RPRS variables. It has been demonstrated that intelligence is an important determinant of the RPRS score with only small $\mathrm{m}$ as a newly pure nonintellective variable. However, the intellectual dimension of FM is not easily pinpointed by verbal or performance intelligence. FM is 
described as an index of the handling of stress tolerance and the degree of comfort or discomfort a person feels with regard to drive impulses. The WAIS Comp subscale seems to cover the FM aspect with, regard to compulsive behavior. The basic assumption of the Comp subtest is the ability to analyze and justify the reasons for certain customs and the ability to act in conformity, which are considered to be important elements of intelligent behavior in our society. The WAIS variables which are the best measures of stress tolerance are A, DS, D sym, the anxiety triad. Low scores on a11 these subscales suggest trait anxiety. These subscales have low loadings on the RPRS factor to which FM is the main contributor. High scores on FM are not significantly. correlated with high or low scores on A, DS, D sym. One might hypothesize that a certain amount of anxiety enhances the effectiveness of psychotherapy, but that too much anxiety immobilizes the person. Thus, anxiety would have a curvilinear relationship to success in therapy. Factor analysis, because it is based on correlation, assumes a linear relationship. Therefore, no conclusions about the relationship between $F M$ and $A, D S, D$ sym can be made in this study. It might also be pointed out that previous research correlating the RPRS subscores with success in therapy (see Table I) has failed to find positive correlations between FM and success in therapy. Therefore, further research examining the curvilinear relationship between FM and success in therapy seems to be indicated.

The Information subscale seems to be the best measure of the verbal intelligence dimension present in the RPRS. Whether D sym can 
be used as a measure of the performance intelligence present in the RPRS needs to be explored in future research. It was mentioned before that for the sample used in this study the intercorrelations of D sym with the other WAIS subscales are considerably lower than the intercorrelations given in the WAIS manual. Hybl and Stagner (1952) found that the D sym subtest from the Wechsler-Bellevue was a good prediction of client improvement. Whether I, D sym, Comp and possibly A, DS, D sym can be used as predictors of success in therapy warrants further research. Finally, the reasons for the ambiguity of the status of $C$ in this study should be explored and pursued. That color responses in general are not easy to interpret has been pointed out by Frank in his 1976 review of the literature linking color responses to affect. Frank states that there are too many confounding aspects in the use of color (hue, location, saturation, individual color preference) to generalize the meaning of color responses.

This study has shown that a relationship exists between intelligence and the RPRS. The results also point to future research using certain WAIS subscales as possible predictors for success in therapy. 
BIBLIOGRAPHY

Auerbach, S. M. and Spielberger, C. D. The Assessment of State and Trait Anxiety with the Rorschach Test. Journal of Personality Assessment (1972), Vo1. 36 (3), 314-335.

Barron, Frank. Some test correlates of responses to psychotherapy. Journal of Consulting Psychology, 1953, 17 (4), 235-241.

Berger, J., Marinkouv, M., Stojanovic, L., and Aleksic Z. The levels hypothesis as a theoretical framework for the prediction of outcome in the treatment of psychoneurotics. Psihologija, Sept. 1969, V2 (2) 421-431.

Bloom, B. L. Prognostic significance of the underproductive Rorschach. Journal of Projective Techniques, 1956, 20, 366-371.

Cartwright, R. D. Predicting response to client-centered therapy with the Rorschach Prognostic Rating Scale. Journal of Counse1ing Psychology, 1958, $\underline{5}, 11-15$.

Cattell, R. B. "The Meaning and Strategic Use of Factor Analysis". Handbook of Multivariate Experimental Psychology, R. B. Cattell, (ed.), Chicago: Rand McNally and Co., (1966).

Comrey, Andrew L. A First Course in Factor Analysis. Academic Press: New York and London, 1973.

Edinger, J. D. and Weiss, W. U. The relation between the altitude quotient and adjustment potential. Journal of Clinical Psychology $\cdot(1974), \underline{30}, 510-513$.

Endicott, N. A. and Endicott, J. Prediction of improvement in treated and untreated patients using the RPRS. Journal of Consulting and Clinical Psychology, 1958, 5, 11-15.

Filmer-Bennett, G. The Rorschach as a means of predicting treatment outcome. Journal of Consulting and Clinical Psychology, 1955, $19,331-334$.

Frank, G. On the validity of hypotheses derived from the Rorschach: 1. the relationship between color and affect. Perceptual and Motor Skills, 1976, 43, 411-427.

Garfield, S. L. and Affleck, D. C. An appraisal of duration of stay in outpatient psychotherapy. Journal of Nervous Mental Disorders, $1959,129,492-498$.

Garfield, S. L. and Affleck, D. C. Therapists' judgments concerning patients considered for psychotherapy. Journal of Consulting Psychology, 1961, 25, 505-509. 
Garwood, John. A guide to research on the Rorschach Prognostic Rating Scale. Journal of Personality Assessment, (1977), 41, $117-119$.

Harman, Harry H. Modern Factory Analysis. Chicago: The University of Chicago Press, (1960).

Harris, R. E. and Christiansen, C. Prediction of response to brief psychotherapy. The Journal of Psychology, 1946, 21, 269-284.

Hirt, M. Measurement and the Rorschach in M. Hitt (Ed.), Rorschach Science. Glencoe, I11.: Free Press, 1962, pp. 3-16.

Hollingshead, A. B. and Redlich, F. C. Social Class and Mental Illness: A Community Study. New York: Wiley, 1958.

Hyblar \& Stagner, R. Frustration tolerance in relation to diagnosis and therapy. Journal of Consulting Psychology, 1952, 16, 163-170.

Johnson, E. Z. Klopfer's Prognostic Rating Scale used with Rowen's Progressive Matrices in play therapy prognosis. Journal of Projective Techniques, $1953,17,320-326$.

Kirkner, F. J., Wisham, W. W., and Giedt, F. H. A report on the validity of the Rorschach Prognostic Rating Scale. Journal of Projective Techniques, 1953, 17, 465-470.

Klopfer, B., Ainsworth, M., Klopfer, W. and Holt, R. Developments in the Rorschach Technique. (Vol. 1) New York: Harcourt, Brace and World, 1954.

Mindess, H. Predicting patient's responses to psychotherapy: A preliminary study designed to investigate the validity of the Rorschach Prognostic Rating Scale. Journal of Projective Techniques, 1953, 17, 327-334.

Newmark, C. S., Finkelstein, M., Frerking, R. A. Comparison of the predictive validity of two measures of psychotherapy prognosis. Journal of Personality Assessment, 1974, 38, (2), 144-148.

0'Connell, R. A., Golden, J. M., and Semonsky, C. K. Which adolescent stays in group psychotherapy? Adolescence, 1972, $\underline{6},(25)$, 51-60.

Salzberg, H. C. and Bidus, D. R. Development of a group therapy screening scales, an attempt to select suitable candidates and predict successful outcome. Journal of Clinical Psychology, 1966, 22, (4), 478-481. 
Saunders, D. R. Patterns of intellectual functioning and their implications for the dynamics of behavior. Reprinted from: The Role and methodology of classification in psychiatry and psychopathology. Washington, D.C.: U. S. Department of Health, Education and Welfare, Public Health Service, undated.

Seidel, C. The relationship between Klopfer's Rorschach Prognostic Rating Scale and Phillip's Case History Prognostic Rating Scale. Journal of Consulting and Clinical Psychology, 1960, 24, 46-49.

Sheehan, J., Frederick, C., Rosevaer, W., and Spigelman, M. A validity study of the Rorschach Prognostic Rating Scale. Journal of Projective Techniques, 1954, 18, 233-239.

Wechsler, D. The measurement and appraisal of adult intelligence. Baltimore: Williams and Wilkins, 1958.

Weiss, W., Edinger, J. D. Perceptual and verbal correlated of the Rorschach Prognostic Rating Scale. Journal of Clinical Psychology, 1974,30 (4), 574-576.

Whiteley, J. M. and Blaine, G. B., Jr. Rorschach in relation to outcome in psychotherapy with college students. Journal of Consulting and Clinical Psychology, 1967, 31, 595-599.

Williams, G. T. and Monder, R. A one year concurrent validity study of the Rorschach Prognostic Rating Scale. Journal of Projective Techniques, $1967,31,30-33$.

Zimmerman and Woo-Sam. Clinical Interpretation of the Wechsler Adult Intelligence Scale. Grune and Stratton: New York, San Francisco, London, 1973. 